\title{
Pulsed laser deposition of a dense and uniform Au nanoparticles layer for surface plasmon enhanced efficiency hybrid solar cells
}

\author{
Vincenzo Resta - Anna Paola Caricato - Anna Loiudice • \\ Aurora Rizzo • Giuseppe Gigli • Antonietta Taurino • \\ Massimo Catalano • Maurizio Martino
}

Received: 8 April 2013/Accepted: 17 September 2013/Published online: 6 October 2013

(C) Springer Science+Business Media Dordrecht 2013

\begin{abstract}
A highly dense and uniform layer of $\mathrm{Au}$ nanoparticles (NPs) on poly(3,4-ethylenedioxythiophene):poly(styrenesulfonate) (PEDOT:PSS) film has been produced by the pulsed laser deposition (PLD) technique toward the production of an improved efficiency photovoltaic device. The advantage of PLD over other techniques is the easy and precise control of the Au NPs size and spatial distribution, without needing of further NP surface functionalization. The efficiency enhancement factor related to $\mathrm{Au}$
\end{abstract}

V. Resta $(\square)$

Department of Engineering for Innovation, University of Salento, Cittadella della Ricerca, SS 7 “Appia”, km 706, 72100 Brindisi, Italy

e-mail: vincenzo.resta@le.infn.it;

vincenzo.resta@unisalento.it

A. P. Caricato - A. Loiudice - G. Gigli - M. Martino Department of Mathematics and Physics "E. De Giorgi", University of Salento, Via Arnesano, 73100 Lecce, Italy

A. Loiudice · A. Rizzo · G. Gigli

National Nanotechnology Laboratory (NNL), Istituto Nanoscienze-CNR, Via Arnesano n. 16, 73100 Lecce, Italy

A. Loiudice - A. Rizzo · G. Gigli Center for Bio-Molecular Nanotechnologies (CBN) of IIT@NNL-UniLe-Lecce, Via Barsanti c/o STAMMS, 73010 Arnesano, Lecce, Italy

A. Taurino - M. Catalano Institute for Microelectronics and Microsystems, IMMCNR, Via Monteroni, 73100 Lecce, Italy
NPs doping has been evaluated in a solar cell based on poly-(3-hexylthiophene):[6,6]-phenyl- $\mathrm{C}_{61}$-butyric acid methyl ester (P3HT:PCBM) diffused bilayer. The short-circuit current density, $J_{\mathrm{SC}}$, increases by $18 \%$ and the power conversion efficiency by $22 \%$, respectively, in comparison with an equivalent device without $\mathrm{Au}$ NPs. The optical and morphological properties of the Au NPs layer have been selected in order to evaluate the contribution of the surface plasmon resonance as enhancement factor of the solar cell efficiency, in a range size where light scattering is negligible.

Keywords Hybrid photovoltaics - Metal nanoparticles $\cdot$ Surface plasmon resonance . Pulsed laser deposition - Conjugated polymers . Solar cells $\cdot$ Energy conversion

\section{Introduction}

Devices based on organic photovoltaic (OPV) materials currently represent one of the most intriguing alternatives to inorganic solar cells due to the high versatility of polymers in terms of mechanical properties, simple processability, and cost effectiveness (Gunes et al. 2007). Conjugated polymers have emerged as promising materials, due to their high absorption coefficients in the visible region, allowing capture of suitable solar spectrum intensity with small film thicknesses. Bulk heterojunction (BHJ) 
configuration with a light absorbing polymer (donor) and a soluble fullerene (acceptor), ensures a better absorption/charge collection due to the intermixing between the two components. However, the thickness of the active layer is often limited by the low chargecarrier mobility and short exciton diffusion lengths of most polymers, resulting in the absorption of a small fraction of the incident sun light (Tromholt et al. 2012).

The inclusion of metal nanoparticles (NPs) has been proposed as a possible route to improve the light trapping in the active layer without increasing its thickness that would inevitably affect the device resistance (Chen et al. 2009). The underlying mechanism is still debated, and it has been often attributed to the coherent collective oscillation of conduction electrons surrounding the NPs, i.e., the surface plasmon resonance (SPR), responsible for a selective light extinction as well as an intense optical near field enhancement at the metal/polymer interface (Wu et al. 2011; Yang et al. 2011). Another explanation ascribes the higher performance driven by the metal NPs to an improved exciton dissociation inside the active layer by reducing the recombination rate (Wu et al. 2011), and possibly to a better electrical conductivity by introducing "dopant" levels within the HOMOLUMO gap of the electron donor layer (Kim and Carroll 2005). Finally, metal NPs larger than $20 \mathrm{~nm}$ diameter are believed to act as reflection and scattering centers of light (Kreibig and Vollmer 1994; Link and El-Sayed 2003), thus inducing the same effect as the interface area between the hole transport and the BHJ active layers would be increased and allowing the collection of a larger number of holes due to the higher optical path length (Wang et al. 2011).

A case material for these applications is $\mathrm{Au}$, most likely because the SPR can be tuned from the visible $(\lambda \geq 500 \mathrm{~nm})$ to the near IR range by changing the NPs dimensions, shape, substrate and/or surrounding media (Link and El-Sayed 2003), while for Ag the SPR lies in the near UV to visible range and for the rest of metals in the UV (Kreibig and Vollmer 1994). The control of the shape, dimensions, and number density of the NPs is essential to exploit their optical and electrical properties (Resta et al. 2011; Liz-Marzán 2006). Pulsed laser deposition (PLD) is well known for its capability of producing a finely tailorable, highly dense and uniform layer of metal NPs on a large variety of substrates (Eason 2007; Resta et al. 2006, 2009), by controlling the plasma expansion dynamics via the laser parameters and the chamber configuration (Resta et al. 2009, 2011; Gonzalo et al. 2007). The nucleation and growth occur directly onto the surface of the chosen substrate, then avoiding NPs agglomeration. Consequently, no NPs functionalization is needed as for typical chemical based synthesis techniques, in which the introduction of a capping ligand often interferes with the optical properties of the NPs (Fung et al. 2011; Topp et al. 2010). Moreover the NPs produced by PLD, densely and uniformly cover the substrate (Resta et al. 2006, 2009), whereas the wet deposition methods for colloidal NPs often lead to aggregation and irreproducible coverage (Zhao et al. 2013).

In the present work, we study the effect of the inclusion of Au NPs produced by PLD in the active layer of an OPV cell in diffused bilayer geometry, fabricated by a sequential coating of the donor poly(3-hexylthiophene), (P3HT), and the acceptor [6,6]phenyl- $\mathrm{C}_{61}$-butyric acid methyl ester, (PCBM), from orthogonal solvents. In spite of the solvent orthogonality, this solution-based technique does not lead to a well-defined bilayer with a sharp interface, but it rather gives rise to a graded $\mathrm{BHJ}$, resulting from the diffusion of the PCBM in the P3HT layer (Loiudice et al. 2012a, b). The NPs have been deposited onto the poly(3,4-ethylenedioxythiophene):poly(styrene sulfonate) (PEDOT:PSS) hole transport layer, coating the indium tin oxide (ITO) transparent anode. In such a configuration, the NPs are placed close to the P3HT absorber layer in order to induce a localized SPR within the device structure, exploiting the near-field enhancement, and to minimize the NPs reflection of the incident light before reaching the active layer. The small size of the NPs guarantees that the scattering-toextinction ratio is well below $\sim 10^{-3}$ (Niesen et al. 2012), remaining negligible any contribution from surface roughness and reflection/scattering inside the electron donor layer (Wang et al. 2011).

\section{Experimental}

Synthesis of Au NPs

The Au NPs layer was produced by PLD in vacuum $\left(5 \times 10^{-6}\right.$ mbar $)$ at room temperature on the ITO/ PEDOT:PSS and on the carbon-coated grid/PEDOT:PSS substrates placed in front of the target. 
The distance from the target was $40 \mathrm{~mm}$, and the substrates were kept in motion during the deposition, in order to obtain a homogeneous and uniform covering by the NPs (Gonzalo et al. 2007). The deposition was carried out by focusing an ArF laser beam $[\lambda=193 \mathrm{~nm}, \tau=20 \mathrm{~ns}$ full width half maximum (FWHM)] to a spot of $1.1 \pm 0.1 \mathrm{~mm}^{2}$ on a $99.99 \% \mathrm{Au}$ target at an angle of $45^{\circ}$ with respect to the target normal. The laser repetition rate was set at $10 \mathrm{~Hz}$, the fluence at the target site was $\sim 2.5 \mathrm{~J} \mathrm{~cm}^{-2}$ and the number of pulses was 300 .

Device fabrication

The device architecture of the BHJ solar cell is ITO/ PEDOT:PSS/(Au NPs)/P3HT:PCBM/LiF/Al. The solar cells were fabricated on ITO-coated glass substrates, with a sheet resistance of $15 \Omega \mathrm{cm}^{-2}$. The ITO-coated glass substrates were sequentially cleaned by ultrasonication in deionized water, acetone, and 2-propanol, and then cleaned for $10 \mathrm{~min}$ at $85^{\circ} \mathrm{C}$ by using TL-1 solution cleaner. PEDOT:PSS (Stark, CLEVIOS) was spincoated at 4,000 rpm for $60 \mathrm{~s}$ to form a film of $40 \mathrm{~nm}$ thickness and dried at $140{ }^{\circ} \mathrm{C}$ for $15 \mathrm{~min}$ in nitrogen atmosphere (Loiudice et al. 2012a; Park et al. 2009). Under the same conditions, PEDOT:PSS were also deposited on a carbon coated grid, for TEM analyses. After the deposition of the Au NPs, the P3HT (Rieke metals, $<98 \%$ regioregular) solution in chlorobenzene (CB) was spin-casted onto the PEDOT:PSS/Au NPs layer, and was allowed to dry in the glove box for $20 \mathrm{~min}$. Then, the PCBM layer was spin-casted from dichloromethane. Such a procedure gives rise to a graded BHJ structure, where the P3HT:PCBM layer has an overall thickness of $100 \mathrm{~nm}$ (Loiudice et al. 2012a, b). The device fabrication was completed by thermal evaporation of $0.6 \mathrm{~nm} \mathrm{LiF}$ and $100 \mathrm{~nm} \mathrm{Al}$ as the cathode under vacuum at a base pressure of $1.3 \times 10^{-6}$ mbar. For comparison, the device without Au NPs was also fabricated following the same procedure.

\section{Characterization}

The optical characterization of the Au NPs on PEDOT:PSS was carried out by a Perkin Elmer Lambda 900 spectrophotometer in the wavelength range $350-850 \mathrm{~nm}$, with a resolution of $2 \mathrm{~nm}$. The optical extinction spectra of the specimens are determined from the experimentally measured transmission $(T)$ spectra as ln (1/T). Morphological and structural characterization of the Au NPs was performed by Scanning Electron Microscopy (SEM) and Transmission Electron Microscopy (TEM) investigations on the ITO/PEDOT:PSS/Au and grid/PEDOT:PSS/Au samples, respectively. A Zeiss NVISION 40 Focussed Ion Beam system equipped with a high resolution GEMINI electron column was used for SEM and a Leo 922 instrument operating at $200 \mathrm{kV}$ accelerating voltage with a pointto-point resolution of $0.24 \mathrm{~nm}$ was used for the TEM analyses. The $J-V$ characteristics of the device were measured under AM1.5G $100 \mathrm{~mW} \mathrm{~cm}{ }^{-2}$ illumination using a calibrated Spectra Physics Oriel $150 \mathrm{~W}$ Solar Simulator. The photon to current efficiency (IPCE) was measured by using a $300 \mathrm{~W}$ Xe Arc light source coupled to a monochromator to create the scanning light. A dualchannel Merlin lock-in amplifier was utilized for the sensitive optical power and current measurements. The surface roughness of the P3HT/PCBM layer, in the final device with and without Au NPs, was analyzed by means of a Park Systems XE-70 Atomic Force Microscope (AFM).

\section{Results and discussion}

NPs characteristics

The extinction spectrum of the Au NPs on PEDOT:PSS produced by PLD and measured before the P3HT:PCBM covering, is shown in Fig. 1. The optical response is characterized by a broad band related to the

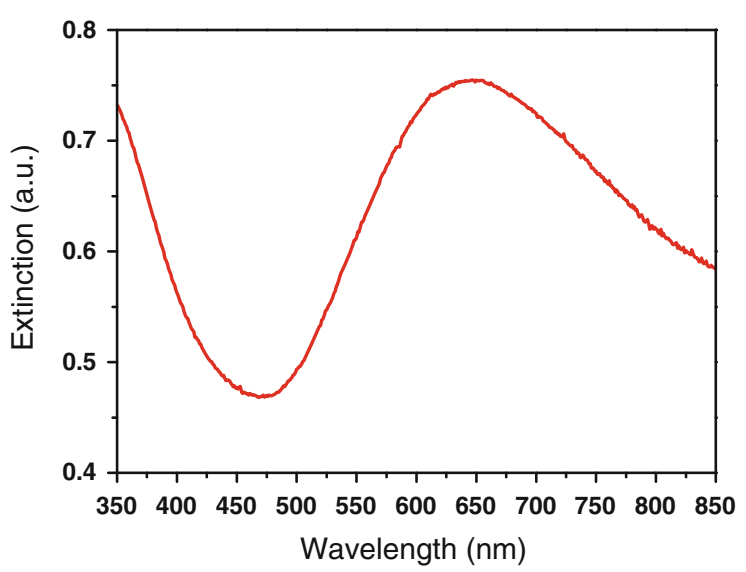

Fig. 1 Extinction spectrum of the Au NPs on PEDOT:PSS measured before the deposition of P3HT:PCBM layer 
SPR at $645 \mathrm{~nm}$, with a FWHM of about $250 \mathrm{~nm}$. On the contrary, the increase of the extinction in the close UV region is mostly due to interband transitions (Resta et al. 2006; Balamuruga and Maruyama 2005).

The morphology characterization of the Au NPs on PEDOT:PSS is reported in Fig. 2, where a $200 \times 200 \mathrm{~nm}^{2}$ SEM image of the sample (Fig. 2a) is shown, demonstrating that the nucleation of the NPs has been obtained uniformly in the substrate surface, and that a highly dense coverage has been achieved. The bright field TEM image of the NPs is in Fig. 2b and the related electron diffraction pattern, in Fig. 2c, shows a series of rings typical of polycrystalline material, whose lattice spacings are compatible with gold (Villars and Calvert 1997). The corresponding Miller Indices are also indicated. The presence of two families of NPs with different diameters is evidenced by the bimodal size distribution of the histogram in Fig. 2d, calculated by studying areas of at least $100 \times 100 \mathrm{~nm}^{2}$. The smallest NPs have an average diameter of $1.8 \pm 0.4 \mathrm{~nm}$, whereas an average value of $5.2 \pm 2.2 \mathrm{~nm}$ was obtained for the largest NPs. The NPs number density has been estimated to be
$1 \times 10^{12} \mathrm{~cm}^{-2}$, taking into account only NPs with diameter $\geq 2 \mathrm{~nm}$, corresponding to a surface filling factor of $29 \%$.

Typical bi-modal distribution of small and large NPs relates to the competition between the implantation and the nucleation processes of the metal atoms into and at the surface of the substrate, respectively (Gonzalo et al. 2005). The implanted layer is commonly located at a depth $<2 \mathrm{~nm}$ from the substrate surface and characterized by a constant gold content normally $\leq 2 \times 10^{15}$ atoms $\mathrm{cm}^{-2}$, irrespective the total amount of gold atoms ejected by the target, and is produced during the growth process in addition to the NPs formed at the surface. It is worth pointing out that in the size range $<2 \mathrm{~nm}$, no SPR is observed for metal NPs produced by PLD, most likely due to the reduced number of electrons contributing to collective oscillations (Requejo-Isidro et al. 2005). Nevertheless, the interlayer dipole interaction cannot be excluded as an enhancement factor of the larger NPs' near field even in spectral regions far from the SPR (Kelly et al. 2003), which is due to dipolar plasmon modes formed on the NPs surface anyway. However, the spatial extent of
Fig. 2 a SEM image of the Au NPs layer on PEDOT:PSS. b Bright field TEM image of the Au NPs on PEDOT:PSS deposited on a carbon coated grid. c Relevant diffraction pattern where the Miller Indices corresponding to the diffraction rings are reported. d Histogram resulting from the statistical analysis of the NPs sizes carried out in areas of at least $100 \times 100 \mathrm{~nm}^{2}$
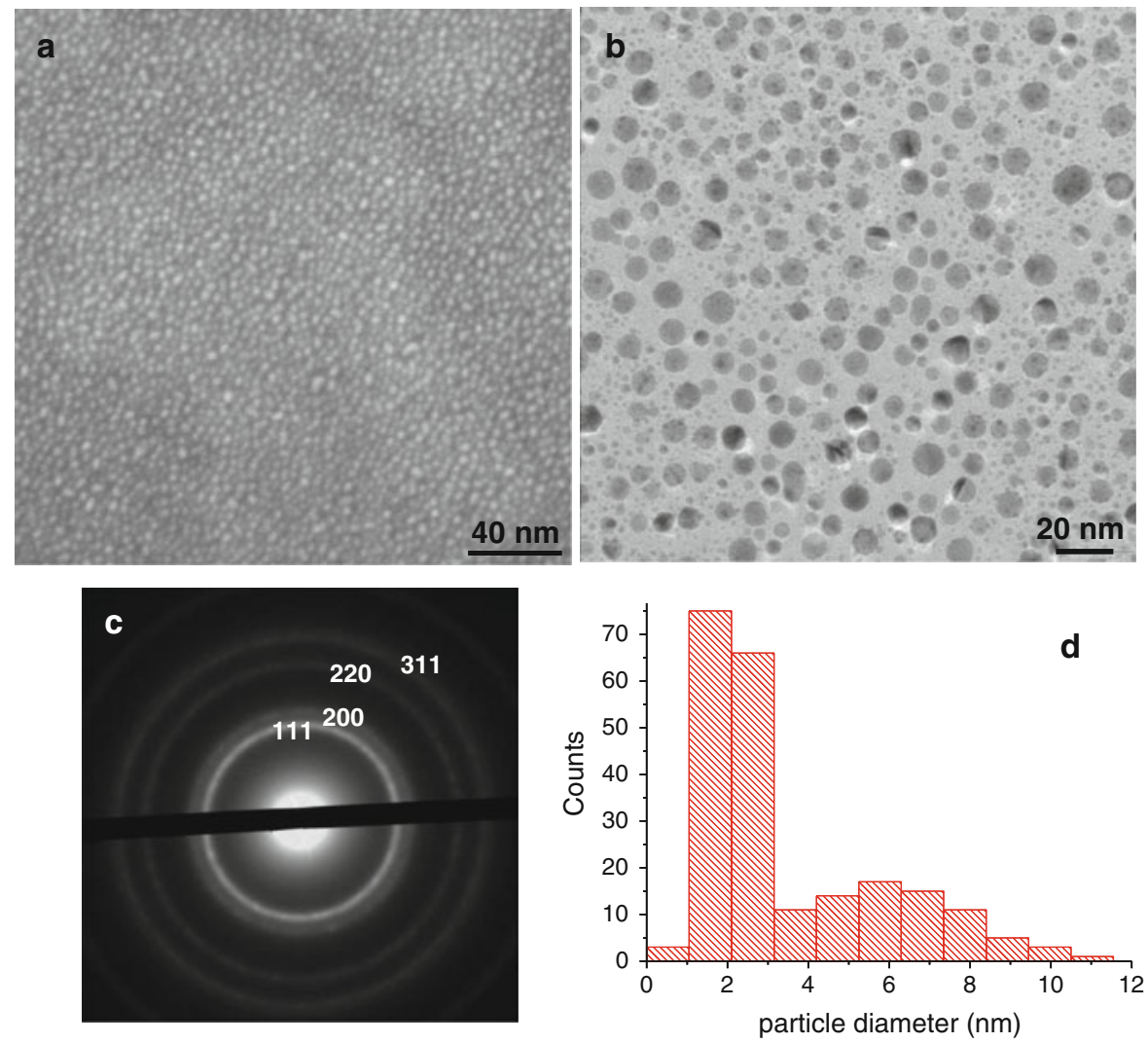
such an absorption enhancement has to be considered not bigger than twice the diameter of the NPs (Niesen et al. 2012; Rand et al. 2004).

\section{Photovoltaic characteristics}

The structure of the hybrid organic-inorganic solar cell is depicted in the inset of Fig. 3, with the NPs located close to the absorber, in order to induce a localized surface plasmon within the device structure, and to utilize its near-field strength as much as possible. The current density $(J)$ versus voltage ( $V$ ) characteristics of the device ITO/PEDOT:PSS/ (Au NPs)/P3HT:PCBM/LiF/Al is shown in Fig. 3, the photovoltaic parameters being listed in Table 1. With the Au NPs between the PEDOT:PSS and the P3HT:PCBM layers, the opencircuit voltage $\left(V_{\mathrm{OC}}\right)$ remains unchanged with respect to the undoped cell, the fill factor (FF) slightly increases from 47 to $49 \%$, and the short-circuit current $\left(J_{\mathrm{SC}}\right)$ increases significantly from 7.57 to $8.96 \mathrm{~mA} \mathrm{~cm}^{-2}$, resulting in an enhancement of $18 \%$. Consequently, the power conversion efficiency (PCE) improved from 1.98 to $2.41 \%$, where the $22 \%$ enhancement was mainly ascribed to the contribution of $J_{\mathrm{SC}}$.

To investigate whether the origin of the $J_{\mathrm{SC}}$ improvement results from the absorption enhancement or not, the extinction spectrum of a solar cell with and without Au NPs was measured, as shown in Fig. 4a. The absorption in the region $350-750 \mathrm{~nm}$ is enhanced by $\sim 20 \%$ for the sample with the Au NPs. The

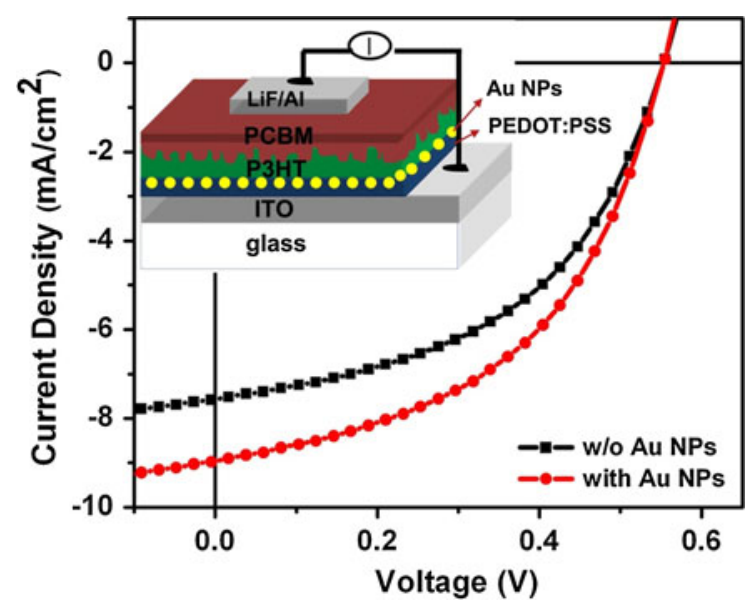

Fig. 3 Comparison of I-V curves of the solar cells with and without Au NPs between the PEDOT:PSS and the P3HT:PCBM layers. The inset shows a sketch of the device configuration incident to photon current efficiency (IPCE) measurements for both solar cells with and without Au NPs are reported in Fig. 4b. An increased IPCE is found for the solar cell with the Au NPs especially in the region between 350 and $500 \mathrm{~nm}$, and also between 550 and $650 \mathrm{~nm}$, fully recovering the absorption spectrum of the Au NPs (Fig. 1) in the close UV as well as SPR band regions. Therefore, it can be safely concluded that the plasmon induced light absorption plays a crucial role in IPCE enhancement, as well as in PCE enhancement, as revealed by the spectral dependent enhancement observed in Fig. 4b. Moreover the AFM on the device without and with Au NPs, as reported in Fig. 4c, d, respectively, shows that no significant changes in device roughness are induced by the deposition of the Au NPs. Indeed, values of 3.7 and $4.6 \mathrm{~nm}$ root-mean-squared roughness, are found for the layer without and with, respectively.

As reported elsewhere, the resonance strength increases when NPs concentration is increased, even when scattering effects cannot be neglected (Wang et al. 2012). The increase observed in $J_{\mathrm{SC}}$ and PCE when $\mathrm{Au}$ NPs are inserted in the P3HT:PCBM layer, respectively, by 18 and $22 \%$, suggests that the influence in the charge transport has to be taken into account, together with the excitation of the SPR. Such an intermediate interpretation between all-optical and all-carrier mobility effects can be justified by considering that our NPs layer is formed by highly dense but small NPs, that can improve carrier mobility without drastically modifying the morphology of the polymer:fullerene blend. The high concentration of small NPs can increase the degree of exciton dissociation, as reported by $\mathrm{Wu}$ et al. (2011), where, however, the effects related to the far-field scattering are not negligible, compared to the absorption due to larger NPs size, namely $\sim 45 \mathrm{~nm}$. In the present case, the surface roughness between PEDOT:PSS and P3HT:PCBM layers is not modified by the NPs, as also indirectly confirmed by noting that the value of FF is not significantly changed with respect to the full OPV device. In facts, it has been reported that a rough P3HT:PCBM surface allows the formation of defect

Table 1 Solar cell performance with and without Au NPs

\begin{tabular}{lllll}
\hline Device & $V_{\mathrm{OC}}(\mathrm{V})$ & $J_{\mathrm{SC}}\left(\mathrm{mA} \mathrm{cm}^{-2}\right)$ & $\mathrm{FF}(\%)$ & PCE (\%) \\
\hline Without $\mathrm{Au}$ & 0.55 & 7.57 & 47 & 1.98 \\
With $\mathrm{Au}$ & 0.55 & 8.96 & 49 & 2.41 \\
\hline
\end{tabular}



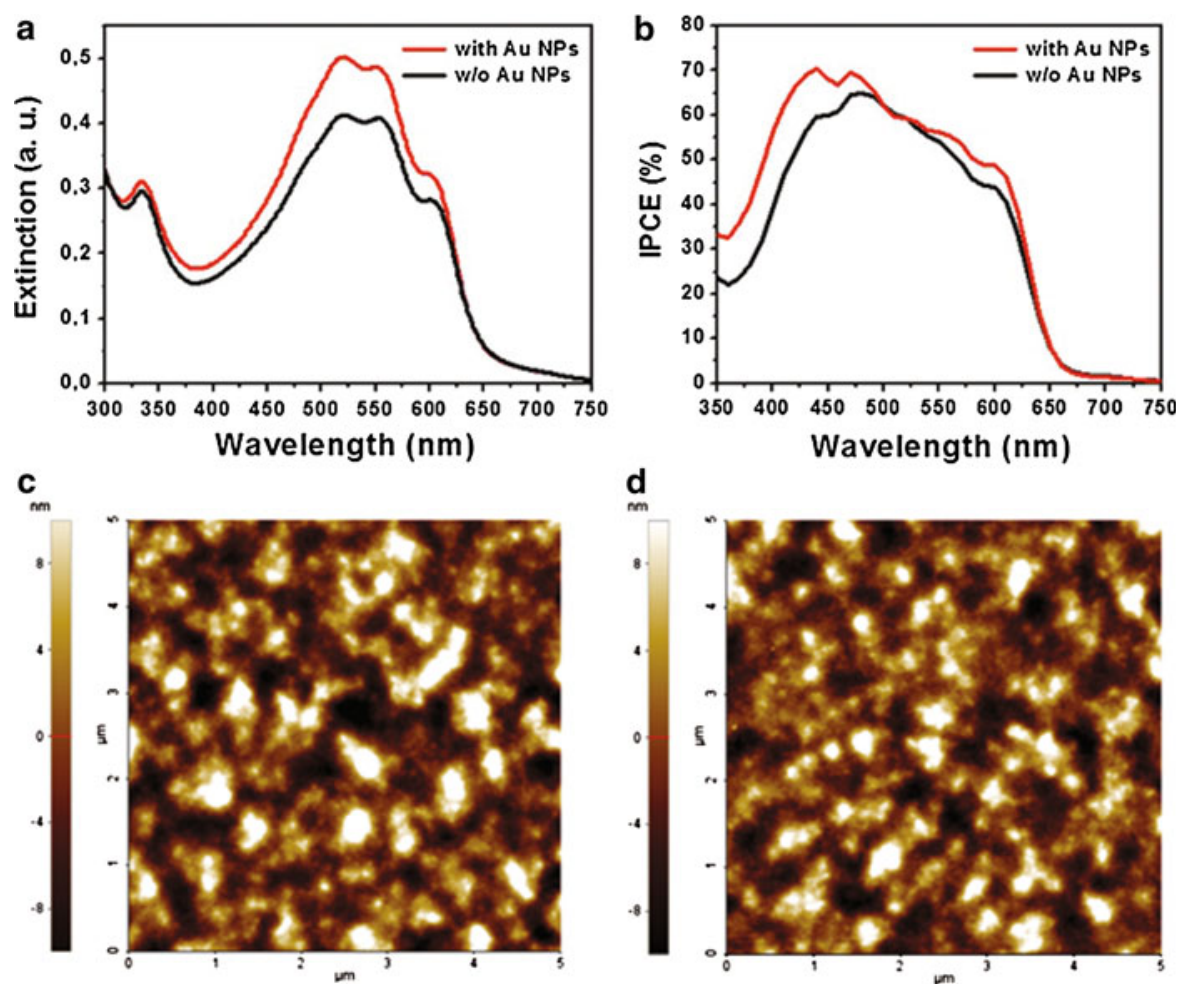

Fig. 4 a Extinction and b IPCE measurements on the devices with and without Au NPs. Topography AFM images of device without (c) and with (d) Au NPs showing

sites improving the exciton dissociation (Fung et al. 2011). However, in the present case no roughness variations are observed as from the AFM images (Fig. 4c, d) and no new recombination centers are introduced, as confirmed by the fact that $V_{\mathrm{OC}}$ value is not modified (Kim and Carroll 2005). Moreover the improved $J_{\mathrm{SC}}$, associated with slightly increased FF and unchanged $V_{\mathrm{OC}}$ with and without NPs, is usually attributed to enhanced absorption of the active layer via strongly enhanced local electromagnetic field in the vicinity of the NPs, leading to an increase in exciton generation (Kim and Carroll 2005; Kim et al. 2008). In addition, accordingly to previous reports $(\mathrm{Wu}$ et al. 2011), the moderate increase of the FF in the device with NPs indicates that the recombination level of geminate exciton is reduced, leading to enhanced degree of exciton dissociation. The NPs morphology chosen in the present case allows to exploit the possible introduction of dopant states within the bandgap of the polymer to provide hopping sites for holes, without affecting the roughness and compromising the charge transport process (Kim and Carroll 2005).

\section{Conclusions}

A hybrid solar cell based on P3HT:PCBM doped with $\mathrm{Au}$ NPs has been produced by depositing the NPs via PLD on a PEDOT:PSS layer before the coating with the active layer. The PCE has been improved by $22 \%$ with respect to the corresponding full OPV device, mainly due to the increase of $18 \%$ on the $J_{\mathrm{SC}}$ and of $4 \%$ on the FF. The PLD technique allowed to produce a highly dense and uniform layer of small NPs, avoiding post-nucleation functionalization and/or thermal annealing procedures. The tailored morphology of the NPs ensures that the reflection/scattering phenomena are negligible, as well as the roughness introduced at the interface between PEDOT:PSS and P3HT:PCBM layers. The optical absorption and the incident to photon current efficiency of the hybrid device increase with respect the OPV, and fully recover the intensity and the spectral evolution of the SPR band related to the Au NPs, thus suggesting that the higher performance is mostly related to SPRinduced field enhancement. 
Acknowledgments This work was supported by the Italian projects Rete Nazionale di Ricerca sulle Nanoscienze ItalNanoNet (FIRB reference number RBPR05JH2P), EFOREnergia da FOnti Rinnovabili (Iniziativa CNR per il Mezzogiorno L. 191/2009 art. 2 comma 44), and by the European project ESCORT-Efficient Solar Cells based on Organic and hybrid Technology (7th FWP-reference number 261920).

\section{References}

Balamuruga B, Maruyama T (2005) Evidence of an enhanced interband absorption in Au nanoparticles: size dependent electronic structure and optical properties. Appl Phys Lett 87:143105

Chen F-C, Wu J-L, Lee C-L, Hong Y, Kuo C-H, Huang MH (2009) Plasmonic-enhanced polymer photovoltaic devices incorporating solution-processable metal nanoparticles. Appl Phys Lett 95:013305

Eason E (2007) Pulsed laser deposition of thin films. Wiley, New York

Fung DDS, Qiao L, Choy WCH, Wang C, Sha WEI, Xiea F, He $S$ (2011) Optical and electrical properties of efficiency enhanced polymer solar cells with Au nanoparticles in a PEDOT-PSS layer. J Mater Chem 21:16349-16356

Gonzalo J, Perea A, Babonneau D, Afonso CN, Beer N, Barnes J-P, Petford-Long AK, Hole DE, Townsend PD (2005) Competing processes during the production of metal nanoparticles by pulsed laser deposition. Phys Rev B $71: 125420$

Gonzalo J, Siegel J, Perea A, Puerto D, Resta V, Galvan-Sosa M, Afonso CN (2007) Imaging self-sputtering and backscattering from the substrate during pulsed laser deposition of gold. Phys Rev B 76:035435

Gunes S, Neugebauer H, Sariciftci SN (2007) Conjugated polymer-based organic solar cells. Chem Rev 107:1324-1338

Kelly KL, Coronado E, Zhao LL, Schatz GC (2003) The optical properties of metal nanoparticles: the influence of size, shape, and dielectric environment. J Phys Chem B 107:668-677

Kim K, Carroll DL (2005) Roles of Au and Ag nanoparticles in efficiency enhancement of poly(3-octylthiophene)/ $\mathrm{C}_{60}$ bulk heterojunction photovoltaic devices. Appl Phys Lett 87:203113

Kim SS, Na S-I, Jo J, Kim D-Y, Nah Y-C (2008) Plasmon enhanced performance of organic solar cells using electrodeposited Ag nanoparticles. Appl Phys Lett 93:073307

Kreibig U, Vollmer M (1994) Optical properties of metal clusters. Springer, Berlin

Link S, El-Sayed MA (2003) Optical properties and ultrafast dynamics of metallic nanocrystals. Annu Rev Phys Chem 54:331-366

Liz-Marzán LM (2006) Tailoring surface plasmons through the morphology and assembly of metal nanoparticles. Langmuir 22:32-41

Loiudice A, Rizzo A, Latini G, Nobile C, de Giorgi M, Gigli G (2012a) Graded vertical phase separation of donor/acceptor species for polymer solar cells. Sol Energy Mat Sol Cells 100:147-152
Loiudice A, Rizzo A, Biasiucci M, Gigli G (2012b) Bulk heterojunction vs. diffused bilayer: the role of device geometry in solution p-doped polymer based solar cells. J Phys Chem Lett 3:1908-1915

Niesen B, Rand BP, Van Dorpe P, Cheyns D, Shen H, Maes B, Heremans P (2012) Near-field interactions between metal nanoparticle surface plasmons and molecular excitons in thinfilms. Part I: absorption. J Phys Chem C 116:24206-24214

Park SH, Roy A, Beaupré S, Cho S, Coates N, Moon JS, Moses D, Leclerc M, Lee K, Heeger AJ (2009) Bulk heterojunction solar cells with internal quantum efficiency approaching $100 \%$. Nat Photon 3:297-303

Rand BP, Peumans P, Forrest SR (2004) Long-range absorption enhancement in organic tandem thin-film solar cells containing silver nanoclusters. J Appl Phys 96:7519

Requejo-Isidro J, del Coso R, Solis J, Gonzalo J, Afonso CN (2005) Role of surface-to-volume ratio of metal nanoparticles in optical properties of $\mathrm{Cu}: \mathrm{Al}_{2} \mathrm{O}_{3}$ nanocomposite films. Appl Phys Lett 86:193104

Resta V, Siegel J, Bonse J, Gonzalo J, Afonso CN, Piscopiello E, van Tendeloo G (2006) Sharpening the shape distribution of gold nanoparticles by laser irradiation. J Appl Phys 100:084311

Resta V, Afonso CN, Piscopiello E, van Tendeloo G (2009) Role of substrate on nucleation and morphology of gold nanoparticles produced by pulsed laser deposition. Phys Rev B 79:235409

Resta V, Gonzalo J, Afonso CN, Piscopiello E, García J (2011) Coverage induced regulation of Au nanoparticles during pulsed laser deposition. J Appl Phys 109:94302

Topp K, Borchert H, Johnen F, Tunc AV, Knipper M, von Hauff E, Parisi J, Al-Shamery K (2010) Impact of the incorporation of $\mathrm{Au}$ nanoparticles into polymer/fullerene solar cells. J Phys Chem A 114:3981-3989

Tromholt T, Madsen MV, Carlé JE, Helgesen M, Krebs FC (2012) Photochemical stability of conjugated polymers, electron acceptors and blends for polymer solar cells resolved in terms of film thickness and absorbance. J Mater Chem 22:7592-7760

Villars P, Calvert LD (1997) Pearson's handbook of crystallographic data for intermetallic compounds, 2nd edn. American Society for Metals, Materials Park (1991) and the desktop edition 1997

Wang DH, Kim DY, Choi KW, Seo JH, Im SH, Park JH, Park OO, Heeger AJ (2011) Enhancement of donor-acceptor polymer bulk heterojunction solar cell power conversion efficiencies by addition of Au nanoparticles. Angew Chem Int Ed 50:5519-5523

Wang CCD, Choy WCH, Duan C, Fung DDS, Sha WEI, Xie F-X, Huang F, Cao Y (2012) Optical and electrical effects of gold nanoparticles in the active layer of polymer solar cells. J Mater Chem 22:1206-1211

Wu J-L, Chen F-C, Hsiao Y-S, Chien CF-C, Chen P, Kuo C-H, Huang MH, Hsu C-S (2011) Surface plasmonic effects of metallic nanoparticles on the performance of polymer bulk heterojunction solar cells. ACS Nano 5:959-967

Yang J, You J, Chen C-C, Hsu W-C, Tan H-R, Zhang X-W, Hong Z, Yang Y (2011) Plasmonic polymer tandem solar cell. ACS Nano 5:6210-6217

Zhao P, Li N, Astruc D (2013) State of the art in gold nanoparticle synthesis. Coord Chem Rev 257:638-665 